\title{
Analysis on key technical nodes of prefabricated concrete construction
}

\author{
Liu Yalong ${ }^{1, *}$ \\ ${ }^{1}$ School of Civil and Traffic Engineering, Shanghai Urban Construction Vocational College, 2360 Jungong Road, Yangpu District, \\ Shanghai, China
}

\begin{abstract}
Prefabricated concrete (PC) buildings are a relatively new type of housing construction. It is not only necessary to ensure the quality of PC components, but also to guarantee the quality of key technical nodes of construction. Based on specific engineering project cases, this article proposes solutions and methods for the key technical nodes of prefabricated concrete construction, and proves their feasibility and effectiveness through practical applications. It illustrates an optimization of prefabricated concrete construction technology to improve construction quality.
\end{abstract}

\section{Introduction}

In recent years, with the rapid development of China's economy the country's construction industry has entered a dynamic stage of development, and is constantly introducing new technologies to improve construction techniques and management methods. The emergence of prefabricated concrete (PC) buildings helps saving resources and energy, and reducing construction pollution. It not only enhances construction efficiency, but also improves construction quality. However, PC structures when compared to the traditional cast-in-place concrete structures have higher construction requirements [1]. Since the size of the precast components cannot be changed, during the construction process the PC structure has relatively high requirements for laying out, measuring accuracy, and the position of reserved holes. If the error exceeds the design range, it will directly affect the installation quality of the component. For example, if the pay-off size is too small, the components cannot be installed, and if the size is too large, it will cause the joints to be too large, too. The accuracy requirements for elevation measurement during construction are also very high, and if the elevation of a shear wall exceeds the allowable error range, it will cause the laminate to fail [2]. Flat installation. In addition, the position and size of the reserved holes in the precast parts must be very accurate, otherwise the grooves need to be re-grooved during the construction process, which will increase the construction difficulty [3]. Therefore, this article analyzes and elaborates on the issues related to the key nodes in the construction of PC buildings.

\section{Problems in key technical nodes of PC construction}

\subsection{Insufficient protection of PC components and serious damage}

The transportation and stacking of $\mathrm{PC}$ components is a prerequisite for quality assurance of PC buildings. As an important part of the prefabricated building construction, the corner board is thin and bulky. If the supporting cushion, transportation fixation, and finished product protection are not applied during the transportation and stacking process, thus it can easily break under the action of external forces. Alternatively, there may be problems such as broken feet or missing corners. In addition, in the process of stacking, if the protection is not strong enough, and the PC components are not stored separately according to the specifications, varieties and stress conditions of the PC components, this will not only cause damage to the components and affect their quality, but will also cause problems such as delays in construction and increased costs.

\subsection{PC components can easily fall off when hoisting, and positioning accuracy is insufficient}

In the construction of PC buildings, there are issues with the lifting position of the PC components and the specifications of the lifting rings. The position of the lifting rings is not rationally determined according to the weight, appearance, and process characteristics of the components themselves, which causes the PC components to fall off easily during lifting. This is particularly visible when hoisting laminated slabs. The prefabricated part of the laminated slab is relatively thin. When the truss steel

\footnotetext{
*Corresponding author's e-mail: liuyalong@succ.edu.cn
} 
bar is randomly used as the hanging point during hoisting, it is easy to drop or damage the laminated slab. In addition, the construction plan did not specify the external wall positioning line and the installation control line, did not consider the space position between the components clearly, and did not clarify the spatial relationship of various steel bars in various connection areas for beams and columns during the construction process. The beam and the main steel bars of the beam collide, resulting in insufficient positioning accuracy during the lifting construction of the PC components, and the splicing installation process is not smooth, which affects the joints of the components, and reduces the construction quality.

\subsection{Connection part of PC component is weak and easy to offset}

The connection of PC components is a key link in the quality assurance of prefabricated buildings. During the construction process, the construction staff confirmed that the key operation has been completed based on the grout left in the grouting hole [4]. However, it is more difficult to inspect the hole, so it is challenging to guarantee the integrity of the key node. If the grouting is not full or the grouting hole is blocked, it will not only make the connection point weak, but also endanger the rigidity and strength of the building and affect its quality. In addition, when the sleeve is connected to each member during construction, the sleeve position and the steel bar position will be offset. If the offset is not large, the rebar can barely be inserted into the reserved hole and will have little effect on the connection of the component. However, if the size difference causes the offset position to be misaligned, the rebar cannot be inserted into the reserved hole, requiring re-manufacturing of the component. In addition, the pipeline design failed to consider the problem of the corner radius, leading to the right angle problem in the preembedded hydropower pipeline in the prefabrication link. The occurrence of these problems increased the difficulty of the on-site wiring construction.

\section{Project example}

This article takes the reconstruction project of the shanty towns in Xiacheng District of Hangzhou as an example to discuss the key technical nodes of PC construction. The construction area of this project is about $364,300 \mathrm{~m} 2$, the plot ratio is 2.9 , and the residential proportion is $70 \%$. This project adopts the PC structures for residential buildings above 4 floors in height, and the PC rate of standard floors can reach $34 \%$. There are a total of four standard apartment designs. Many standard templates were used, and a high copy rate was utilized. The PC components are mainly reinforced concrete corner panels, laminated panels, prefabricated panels, shear walls, prefabricated balconies, and prefabricated stairs, etc. In the construction process of this project, in order to avoid the damage of PC components during transportation and stacking, the falling off during hoisting and the misalignment during connection, suitable measures were adopted for the key technical nodes of the PC construction to obtain a good effect.

\section{Measures and methods of key technical nodes in PC building construction}

\subsection{Strict implementation of design and production specifications}

In the design process of $\mathrm{PC}$ components, designers should continuously communicate with the prefabrication yard, harmonizing the different needs of the construction unit and other aspects, and finally form a design plan of the specific PC component. The design adopts the BIM technology to make the size of the steel frames accurate. The technicians of the prefabrication plant should check and correct the positions of steel bars and embedded parts before concrete pouring. For the accurate size of the reinforced concrete protective layer, special plastic pads or washers can be used as auxiliary tools. For the PC components with small cross-sectional dimensions, small vibrating rods, dense vibration points, and appropriately extended vibrating time are recommended to ensure the quality of PC components. In order to achieve precision in the size of PC components, $\mathrm{PC}$ component manufacturers experiment with intelligent construction methods, such as robotic arms, to avoid product defects caused by human error as much as possible. It is recommended to appoint a dedicated worker to review the appearance quality and size of the finished PC components, and repair defective PC components.

\subsection{Strengthening protection measures to reduce component damage}

In order to avoid collision damage to the PC components during transportation, it is necessary to use a transport rack to fix and keep the PC components as horizontal as possible to avoid shaking and bumping. When storing on site, it is necessary to use the tripod steel channel to make the stacking bracket meets the rigidity requirements, and stack the PC components symmetrically to avoid overturning. The horizontal stacking of the laminated board adopts steel pipes or wooden square blocks to be vertical to the direction of the truss reinforcement, and should not exceed six floors. At the same time, considering that the PC components are prone to deformation and damage during the on-site hoisting process, the spreader should use steel poles as much as possible and be hoisted vertically to effectively prevent deformation. For problems such as rotating plates and fractures that are likely to occur during hoisting, it is recommended to use the L-shaped auxiliary force transfers for the corner plate to the auxiliary spreader, and to control the span of the prefabricated plate within the deflection range of the plate, thereby reducing the probability of breaking due to improper handling. In addition, when hoisting, keeping the positions of the two sides of the components level, and using tools such as corner guards to avoid damage to the side grooves and other locations are recommended. 


\subsection{Choose the appropriate connection method for PC components}

At present, PC components generally adopt two connection methods, namely, wet or dry. The case study project adopts the wet connection method, i.e., the PC components are fully integrated using cast-in-place concrete, which greatly improves the overall load-bearing capacity and seismic strength of the PC structure. The wet connection method can also slightly correct the errors in the installation of the components. The dry connection method can be used for components that do not have a load-bearing function, or have a relatively small load in the structure, such as the secondary beams in buildings and the connections between beams, columns, and floors. In other words, the components are connected by bolting or welding, and the PC components arranged at the ends and edges can be connected by dry connection methods.

\subsection{Using BIM and other technologies to ensure accuracy of hoisting}

Through the combination of the BIM and RFID technologies, this project has essentially realized the quality control of the whole prefabrication process, and through the application of 3D laser scanning technology combined with BIM technology, the construction process was verified to conform strictly to the lifting specifications [5]. The preliminarily corrected construction operation sequence ensures that the resultant force of the lifting points coincides with the center of gravity, thus ensuring the assembly progress and quality. When hoisting PC components, horizontal PC components are hoisted at 4 points when the length is less than $4 \mathrm{~m}$. If the components are longer than $6 \mathrm{~m}$, they are hoisted at 6 points. The corresponding position of the hook is equipped with stiffeners and marked by a clear hook mark. When the hook is lifted, the force of each lifting point is even. After hoisting, the level and verticality of PC components should be adjusted repeatedly. The flatness is the foundation of the wallboard mortar. At the same time, the material loss is increased. The new sag sensor ruler can be connected to smart devices through specially configured wireless transmission system, and can realize remote sag adjustment, with a detection accuracy in access of $1 / 1,000$, which greatly improves the wall quality to ensure useful range and accuracy of face perpendicularity detection. In view of the problem of the laminated board falling off during the hoisting process, the reinforcement can be directly carried out around the embedded parts during construction, which not only saves the embedded parts, but also increases the safety factor of the laminated board hoisting process, and improves the construction quality of the prefabricated building. This will make a substantial difference.

\subsection{Cleaning connection before sleeve grouting}

During on-site grouting construction, it is very important to follow strictly the steps of plugging (grouting) $\rightarrow$ plugging the lower drain grouting hole $\rightarrow$ mixing grouting $\rightarrow$ slurry detection $\rightarrow$ pressure grouting $\rightarrow$ plugging the upper drain grouting hole $\rightarrow$ leaving the test block. When grouting, the measurement and positioning of the PC component is very important, and the vertical forcebearing component must ensure that the position, elevation, and verticality of the component are accurate. Before grouting, it is necessary to clean the connecting parts of the components. It is important to ensure that there is no debris, oil, or water in the steel sleeve. Only after the adjustment of the PC component is completed, the positioning is firm, grouting can be carried out. Special reminder is that when we see the slurry hole continuously flowing out of the columnar slurry, we can use the plug to plug it. In order to ensure the quality of the connection, the components after grouting should be protected from shaking or other shocks.

\section{Conclusions}

The prefabricated building construction has transformed the original complicated on-site manual production into the mechanized assembly line mass production of the PC yard. The quality and size of various components are guaranteed. The prefabricated building construction reduces construction waste and environmental pollution, make building construction more energy-efficient and environmentally friendly, and facilitates intelligent transformation.

\section{Reference}

1. Chang-Quan H E , Zong-Zhi D , Su-Feng W . (2015) Path Selection and Development Measure of Construction Industrialization on Assembly Type PC Structure[J]. Journal of Bengbu University

2. Zhengxing G. (2014) Thinking on Generalization and Application of New Prefabricated Concrete Structure[J]. Construction Technology

3. Code for seismic design of buildings (GB 500112010). Beijing: China Construction Industry Press (2010)

4. Li Z, Qi Y, Teng J. (2020) Experimental investigation of prefabricated beam-to-column steel joints for precast concrete structures under cyclic loading[J]. Engineering Structures, 209:110217

5. Shijue Y, Jinyuan L, Renjie H, et al. (2014) Application Study on BIM Technology in Prefabricated Concrete Structure Construction of Senile Apartment [J]. Construction Technology, 36(9): 872-9 